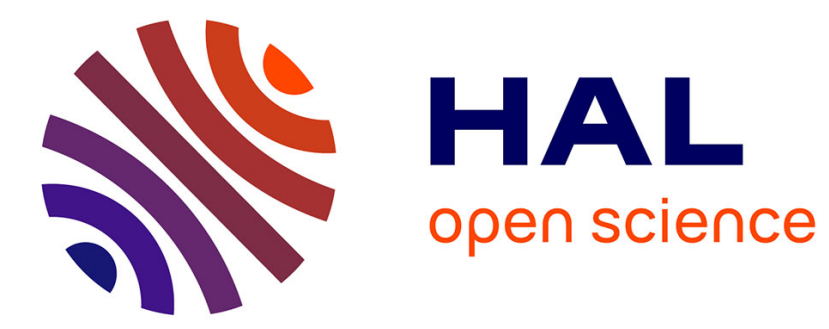

\title{
Orientational glasses. I. A random compressible model for $\mathrm{KCN}-\mathrm{KBr}$
}

\author{
Serge Galam
}

\section{To cite this version:}

Serge Galam. Orientational glasses. I. A random compressible model for KCN-KBr. Journal de Physique I, 1991, 1 (8), pp.1195-1208. 10.1051/jp1:1991200 • jpa-00246405

\section{HAL Id: jpa-00246405 https://hal.science/jpa-00246405}

Submitted on 1 Jan 1991

HAL is a multi-disciplinary open access archive for the deposit and dissemination of scientific research documents, whether they are published or not. The documents may come from teaching and research institutions in France or abroad, or from public or private research centers.
L'archive ouverte pluridisciplinaire HAL, est destinée au dépôt et à la diffusion de documents scientifiques de niveau recherche, publiés ou non, émanant des établissements d'enseignement et de recherche français ou étrangers, des laboratoires publics ou privés. 
Classification

Physics Abstracts

$64.60 \mathrm{~A}-64.60 \mathrm{C}-64.70 \mathrm{P}$

\title{
Orientational glasses. I. A random compressible model for $\mathrm{KCN}-\mathrm{KBr}$
}

\author{
Serge Galam \\ Département de Recherches Physiques, Tour 22-E3, Université Pierre et Marie Curie, 75252 \\ Paris Cedex 05, France
}

(Received 15 March 1991, revised 2 April 1991, accepted 23 April 1991)

\begin{abstract}
Résumé. - Le diagramme de phase des mélanges $\mathrm{K}(\mathrm{CN})_{x} \mathrm{Br}_{1_{-x}}$ est obtenu par une étude de champ moyen d'un modèle ferroélastique compressible anharmonique, aléatoire et dilué. L'intégration des degrés de liberté élastiques produit un modèle non linéaire de percolation. En parallèle, une simple hypothèse linéaire est supposée pour introduire une densité effective des cyanures, afin de tenir compte de leur forme asymétrique par rapport à la forme sphérique des bromes. La percolation de site est ainsi différenciée de la percolation d'orientation correspondante. Trois valeurs caractéristiques dans la concentration de cyanures $x=0,2,0,5$ et 0,6 sont obtenues. Pour $x<0,6$ la transition ferroélastique est perdue. Dans l'intervalle $0,5<x<0,6$, à basses températures les cyanures forment des domaines ferroélastiques avec des orientations aléatoires. Quand $0,2<x<0,5$ il y a un verre collectif dynamique. Il se transforme dans un état d'ions isolés pour $x<0,2$. Il est ensuite montré que le diagramme de phase correspondant, pour les mélanges $\mathrm{K}_{x}\left(\mathrm{Na}_{1_{-}}\right) \mathrm{CN}$ est d'une nature physique différente. Il est lié en particulier à des interactions compétitives aléatoires. Une revue critique du modèle de champ aléatoire orientationnel est présentée. Les résultats fournissent un cadre unifié et consistant pour différents résultats expérimentaux.
\end{abstract}

\begin{abstract}
The full phase diagram of $\mathrm{K}(\mathrm{CN})_{x} \mathrm{Br}_{1-x}$ mixtures is obtained using a mean-field treatment of a dilute random anharmonic compressible ferroelastic model. Integration of elastic degrees of freedom leads to a non-linear percolating problem. No random competing interactions are found. In parallel a simple linear hypothesis for an effective density of cyanides is assumed to account for the asymmetric shape of the cyanide versus the spherical bromide shape. Site percolation of cyanides is thus discriminated from the corresponding orientational percolation. Three characteristic values of cyanide concentration $x=0.2,0.5$ and 0.6 are obtained. For $x<0.6$ the ferroelastic transition is lost. In the range $0.5<x<0.6$, at low temperatures, cyanides form ferroelastic domains with random orientations. When $0.2<x<0.5$ a collective dynamical glass is obtained. It turns to a single ion state for $x<0.2$. It is then shown that the corresponding phase diagram of $\mathrm{K}_{x}\left(\mathrm{Na}_{1-x}\right) \mathrm{CN}$ mixtures is of a different physical nature. It involves in particular random competing interactions. A critical review of the orientational random field model is presented. The results are found to provide a unified and consistent framework to various experimental data.
\end{abstract}




\section{Introduction.}

The existence of an orientational glass state in $\mathrm{K}(\mathrm{CN})_{x} \mathrm{Br}_{1-x}$ mixtures which might be connected to spin glasses has generated a great deal of experimental [1-6], numerical [7] and theoretical work [8-10] in recent years.

Pure KCN undergoes a ferroelastic transition from cubic to orthorhombic symmetry. Site dilution of cyanides with bromides makes the transition to disappear below some critical cyanide concentration $x_{\mathrm{c}} \simeq 0.6$. In the range of concentrations $x<0.6$ the system remains cubic at low temperatures. Moreover the elastic shear constant exhibits a round minimum and diffraction experiments reveal the appearance of a central peak at a so-called freezing temperature [1]. Molecular dynamics calculations have confirmed these results [7]. Although these experimental features have been associated with a non-zero spin glass order parameter [8], most experimental data have been interpreted in terms of a random field model [9]. The associated phase diagram is shown in figure $1[2,4,9]$.

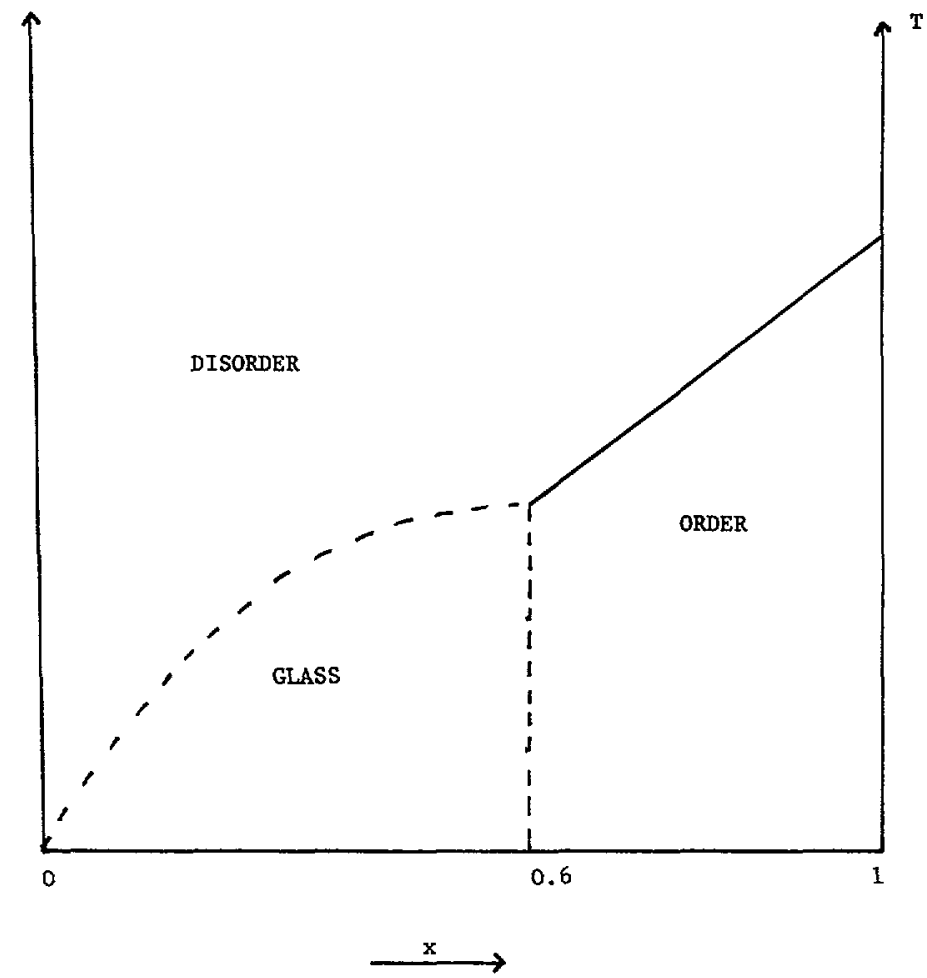

Fig. 1. - Phase diagram from references $[2,4,9]$.

In parallel two characteristic values of cyanide concentration were found in different experiments $[3,6]$. Low frequency shear response measurements indicated an overlap of two elastic loss peaks at $x=0.2$ using the resonance of a torsion pendulum [3]. More recently shear torque measurements suggested the existence of a peculiar glassy-regime in the range $0.5<x<0.6[6]$, thus introducing the particular value $x=0.5$ in the phase diagram, in addition to the values $x=0.2$ and $x=0.6$.

In this paper we first present a microscopic model to describe $\mathrm{KCN}-\mathrm{KBr}$ mixtures [10]. A dilute compressible ferroelastic system is considered. Random anharmonic elastic terms are 
added to the Hamiltonian to account for site fluctuations of bromides [11]. It is then shown that integration of elastic degrees of freedom leads to an effective Hamiltonian of a dilute system with a cyanide-cyanide coupling of the form $J_{0}-A(1-x)$ where $J_{0}$ is the bare initial coupling and $A$ is a constant. The problem is thus found to reduce to a non-linear site percolation problem on a fcc lattice [10]. The associated mean field phase diagram has only one ordered phase and the transition is lost at the percolation threshold given by $p_{\mathrm{c}} \simeq 0.2[12]$.

We then suggest the idea of inhomogeneous steric hindrances in a percolating lattice to introduce the effect of shape difference between cyanides and bromides which are respectively dumbell-like and spherical. This means in particular to discriminate between cyanide site percolation and the corresponding orientational percolation. It is indeed consistent with the discrepancy between the percolation threshold $p_{\mathrm{c}} \simeq 0.2$ and the experimental finding, $x_{\mathrm{c}}=0.6$, at which the ferroelastic transition is lost. On this basis a simple linear formula is assumed for an effective density of free cyanides to implement the phase diagram associated to the random anharmonic compressible model. A sketch of the full phase diagram of $\mathrm{K}(\mathrm{CN})_{x} \mathrm{Br}_{1-x}$ mixtures is obtained and shown in figure 2 . The three

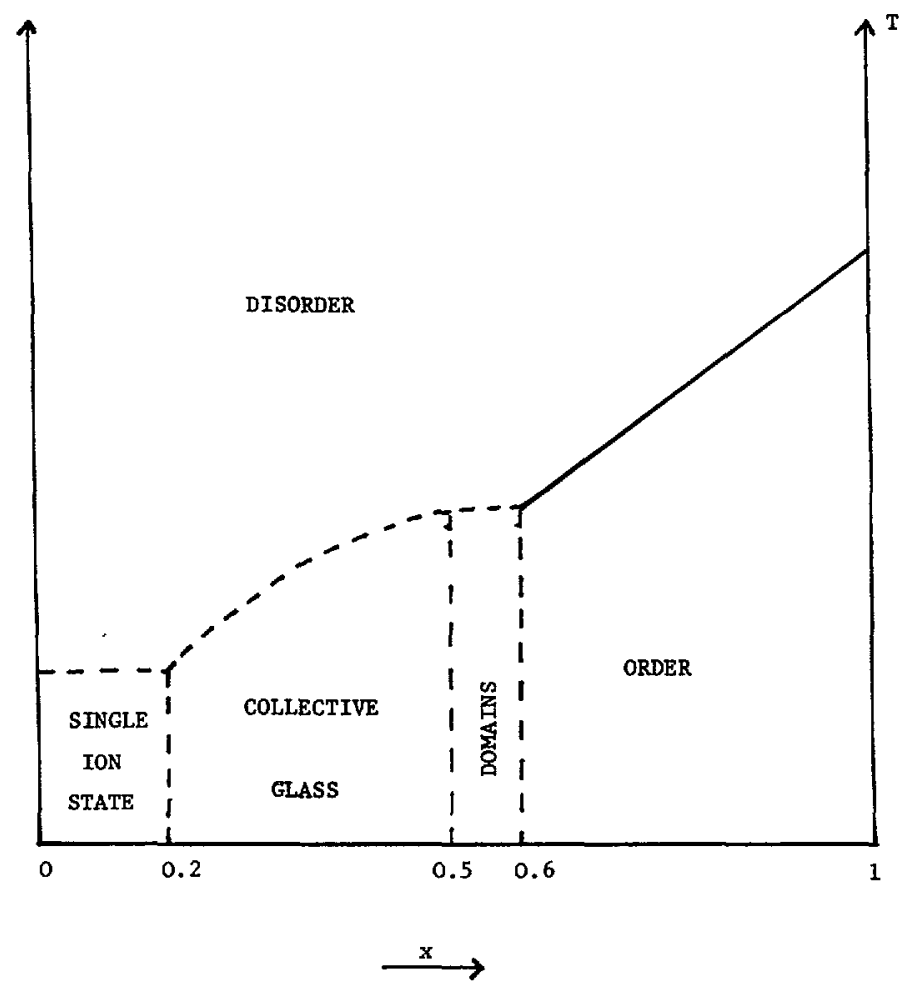

Fig. 2. - Phase diagram proposed in the present work.

experimental characteristic values of concentration, $x=0.2,0.5$ and 0.6 , are found to be related by this formula as thresholds in the phase diagram. Various experimental results [3, 6] are thus interpreted in a unified and consistent framework. The connection to NaCN-KCN mixtures is discussed. Random competing interactions are found to be instrumental in this case while not existing for $\mathrm{KCN}-\mathrm{KBr}$ mixtures. 
This paper is organized as follows. The random anharmonic compressible model used to describe $\mathrm{KCN}-\mathrm{KBr}$ mixtures is presented in section 2. A mean field treatment is performed in part 3. Three possible phase diagrams resulting from this model are described in section 4. The idea of broken percolation is introduced in part 5 to implement the full phase diagram of $\mathrm{KCN}-\mathrm{KBr}$ mixtures. The results are discussed with respect to the experimental situation. Section 6 is devoted to the connection to the glassy phase of $\mathrm{KCN}-\mathrm{NaCN}$ mixtures. A critical review of the orientational random field model is presented in section 7 . It is shown that this model is indeed not allowed by symmetry. Its connection with some experimental results is discussed. A summary of main results of the present work is given in part 8 . Concluding remarks are contained in the last part.

\section{The random anharmonic compressible model.}

In pure KCN molecular crystals, cyanides form a fcc lattice. Dealing with site substitution of $\mathrm{CN}$ with bromides only, we can ignore the interpenetrated alkali lattice. We thus consider a fcc lattice to describe the low temperature part of the phase diagram of $\mathrm{K}(\mathrm{CN})_{x} \mathbf{B r} \mathbf{r}_{1-x}$ mixed crystals. A variable $\varepsilon_{i}$ is associated with each site $i$. It is equal to either one or zero if the site is occupied by respectively a cyanide or a bromide. The $\left\{\varepsilon_{1}\right\}$ are quenched and randomly distributed. The configurational average $\bar{\varepsilon}_{i}$ is equal to the cyanide concentration $x$.

Rotational degrees of freedom of asymmetric dumbell-shaped $\mathrm{CN}$ molecules are represented by classical Heisenberg spin variables $\left\{\mathbf{S}_{i}\right\}$. Elastic vibrations are also introduced to account for translation-rotation couplings.

On this basis we start with a diluted compressible ferromagnetic spin Hamiltonian

$$
H_{0}=-\sum_{(i, j)} J_{i j} \varepsilon_{i} \varepsilon_{j} \mathbf{S}_{i} \mathbf{S}_{j}+\sum_{\langle i, j\rangle} \varphi_{i j}
$$

where $J_{i j}$ is a positive ferroelastic coupling between nearest neighbors $\mathbf{S}_{i}$ and $\mathbf{S}_{j}$, $(i, j)$ denotes a sum over nearest neighbors and $\varphi_{l j}$ is an harmonic elastic potential.

At this stage to make the calculations tractable we choose to consider a simple elastic model. Lattice vibrations are introduced along decoupled one-dimensional chains. Then at first order in the displacements $u_{i}$ of ion $\mathrm{CN}$ or $\mathrm{Br}$ at site $i$, with respect to the pure $\mathrm{KCN}$ lattice, we have

$$
J_{i j}=J_{0}-\left(u_{i}-u_{j}\right) J
$$

and

$$
\varphi_{i j}=\frac{1}{2} \varphi\left(u_{i}-u_{j}\right)^{2}
$$

where $J_{0}, J$, and $\varphi$ are positive constants, and $\langle i, j\rangle$ belong to the same chain. The $\left\{u_{i}\right\}$ are scalars.

However Hamiltonian (1) includes only the non-orientational character of spherical bromides. To account for the difference in volume [11] between $\mathrm{CN}$ and $\mathrm{Br}$ molecules we first consider a mean effect through a uniform anharmonic term,

$$
H_{\mathrm{U}}=-\sum_{\langle i, j\rangle} 2 h_{\mathrm{L}}(1-x)\left(u_{i}-u_{j}\right)
$$

where $h_{\mathrm{L}}$ is associated with long range elastic interactions. On top of this homogeneous dilatation we must ad-1 Jom anharmonic terms to equation (1) to account for the random 
distribution of bromides. At first approximation assuming a spherical shape for cyanides [9], fluctuations in concentration of bromides produce static strains given by a term [11],

$$
H_{\mathrm{A}}=-\sum_{\langle, j\rangle} h_{i j}\left(u_{i}-u_{j}\right)
$$

where

$$
h_{i j}=h_{\mathrm{S}}\left\{\left(2-\varepsilon_{i}-\varepsilon_{j}\right)-2(1-x)\right\}
$$

and $h_{\mathrm{S}}$ is the force resulting from the short range elastic effects due to the difference in volume between assumed spherical $\mathrm{CN}$ and $\mathrm{Br}$ molecules. From equation (5) random static strains can take the following values

$$
2 h_{\mathrm{S}}(x-1), h_{\mathrm{S}}(2 x-1), 2 h_{\mathrm{S}} x
$$

with respective probabilities $x^{2}, 2 x(1-x)$ and $(1-x)^{2}$. The first and last values are, respectively, negative and positive, the middle one being negative for $x<\frac{1}{2}$ and positive for $x>\frac{1}{2}$.

Considering the total Hamiltonian $H_{\mathrm{T}}=H_{0}+H_{\mathrm{U}}+H_{\mathrm{A}}$ it is possible to integrate out elastic degrees of freedom in the pressure ensemble using the Hamiltonian

$$
\tilde{H}=H_{\mathrm{T}}+p \sum_{\langle, J\rangle}\left(u_{i}-u_{j}\right)
$$

where $p$ is an external pressure. Noticing that for decoupled one-dimensional chains the last term of the right hand side of equation (7) is equal to the change in the chain length $\Delta L$, we have the partition function

$$
\dot{Y}=\int \mathrm{d} L \exp (-\beta p \Delta \dot{L}) \int \prod_{2} \mathrm{~d} u_{\imath} \underset{\left\{\mathrm{S}_{1}\right\}}{\operatorname{Tr}} \exp (-\beta H)
$$

where $\beta=1 / k_{\mathrm{B}} T, k_{\mathrm{B}}$ is the Boltzmann constant and $T$ the temperature. Performing a Gaussian integral in $\left\{u_{i}\right\}$, and writing the partition function in the form

$$
Y=\underset{\left\{S_{1}\right\}}{\operatorname{Tr}} \exp \left(-\beta H_{\mathrm{R}}\right),
$$

we define an effective Hamiltonian $H_{\mathrm{R}}$. At zero external pressure we find

$$
H_{\mathrm{R}}=-\sum_{\langle i, J\rangle} G_{i j} \varepsilon_{i} \varepsilon_{j} \mathbf{S}_{i} \mathbf{S}_{j}-\frac{J^{2}}{2 \varphi} \sum_{(i, j)} \varepsilon_{i} \varepsilon_{j} \mathbf{S}_{i}^{2} \mathbf{S}_{j}^{2}
$$

where some constant have been discarded, and

$$
G_{i j}=J_{0}-\frac{J}{\varphi}\left\{2 h_{\mathrm{L}}(1-x)-h_{\mathrm{S}}\left(\varepsilon_{i}+\varepsilon_{j}-2 x\right)\right\},
$$

is a random site coupling. However in equation (10), the product $G_{i j} \varepsilon_{i} \varepsilon_{j}$ can be rewritten as

$$
G_{\imath \jmath} \varepsilon_{i} \varepsilon_{j}=G \varepsilon_{i} \varepsilon_{j}
$$


where

$$
G=J_{0}-\frac{2 h J}{\varphi}(1-x)
$$

and $h=h_{\mathrm{L}}-h_{\mathrm{S}}$. To obtain equation (12) the properties $\varepsilon_{t}^{2}=\varepsilon_{\imath}$ and $\left(\varepsilon_{l}+\varepsilon_{j}\right) \varepsilon_{l} \varepsilon_{\jmath}=2 \varepsilon_{t} \varepsilon_{J}$ have been used. From equation (13) it is seen that $G$ is indeed not random.

It is worth noticing that integration of elastic degrees of freedom from $H_{0}$ instead of $H_{\mathrm{T}}$ would also produce equation (10) with however $G=J_{0}$, i.e., $h=0$ in equation (13). It shows that the net effect of the random distribution of volume differences is to renormalize ferroelastic couplings (Eq. (13)). The random character of the problem reduces to a nonlinear site dilution problem. There exist no random competing interactions.

The harmonic compressible part of the model results in the last term of equation (10). For Ising variables this term is a constant and has no effect. However for other type of variables, for instance spin one, it can turn the transition first order [13]. Moreover it is worth stressing that a more realistic model of elasticity which includes shear forces produces an additional tetralinear term which is long range. In that case the transition seems to be first order always, even for Ising variables [14]. Therefore, to consider Ising variables to obtain the phase diagram associated with equation (10) makes the analysis simple still preserving the main physical features of the problem. A more realistic approach will only change the order of the transition from continuous to first order. Our results should be then taken only qualitatively. From now on we are considering Ising variable $S_{i}$ to describe rotational degrees of freedom.

\section{The bare phase diagram.}

We now perform a mean field treatment of equation (10) to descrite the plastic-solid transitions in $\mathrm{KCN}-\mathrm{KBr}$ mixtures. The corresponding equation of state is given by

$$
\left\langle S_{\imath}\right\rangle=x \operatorname{th}\left\{12 \beta G\left\langle S_{i}\right\rangle\right\}
$$

where 12 is the coordination number on a fcc lattice and $\left\langle S_{i}\right\rangle$ is the orientational order parameter.

From equation (14) it is found that there exists one ordered phase with a continuous transition from the disordered phase at a line given by

$$
k T_{\mathrm{c}}=12 \times\left[J_{0}-\frac{2 h J}{\varphi}(1-x)\right]
$$

where $k$ is the Boltzman constant. The phase diagram is shown in figure 3. Improvement of the model would only make the transition first order and thus shift it towards lower temperatures.

From equation (15) it is seen that to keep temperatures positive the phase diagram of figure 3 holds only as long as $\varphi J_{0}>2 h J$. Otherwise the ordered phase disappears before $x=0$ at a critical concentration

$$
\bar{x}_{c}=1-\frac{\varphi J_{0}}{2 h J}
$$

at which $k T_{\mathfrak{c}}=0$ (Eq. (15)) as shown in figure 4 .

When $\varphi J_{0}<2 h J$, that is for $\bar{x}_{\mathrm{c}}>0$, the present analysis shows that two phase diagrams are indeed possible in the range $x<\bar{x}_{\mathrm{c}}$ :

(i) At $x=\bar{x}_{c}$, the effect of random distribution of volume differences has canceled the 


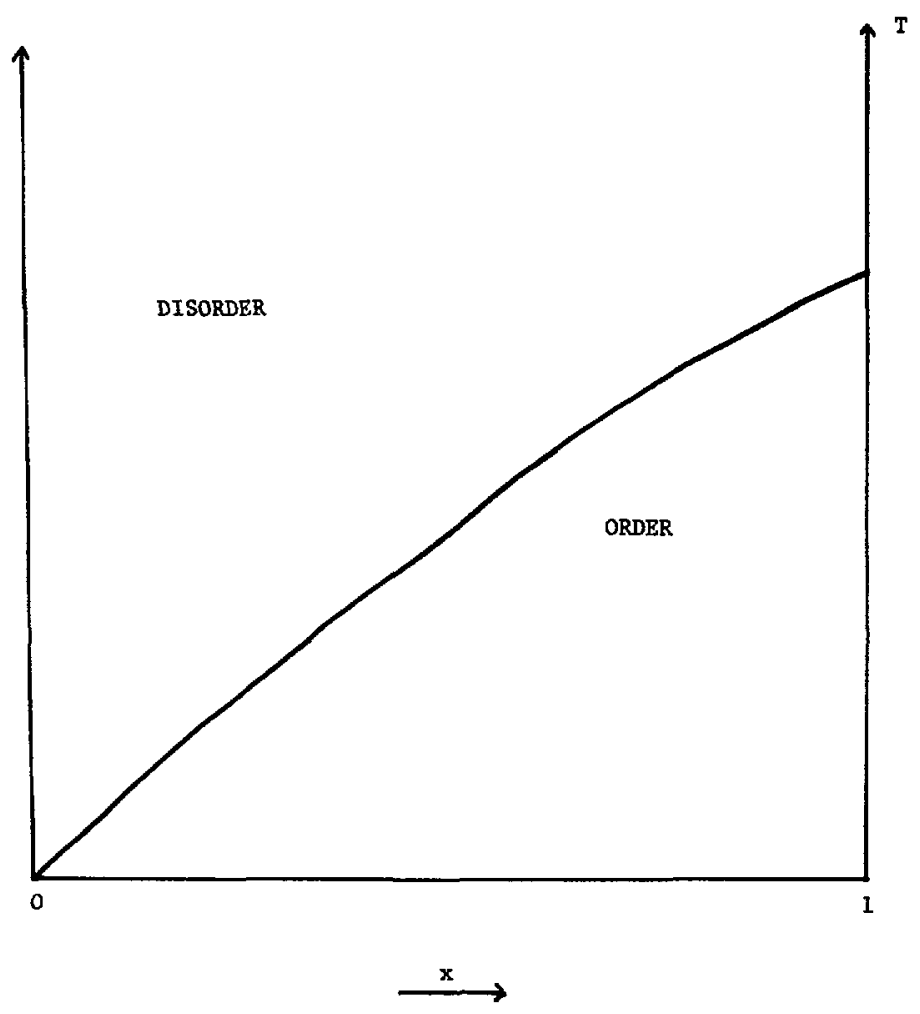

Fig. 3. - Phase diagram without the percolation dressing.

interactions giving $G=0$. For $x<\bar{x}_{\mathrm{c}}$ equation (15) is not valid and thus we assume $G$ stay equal to zero. Rotational degrees of freedom of cyanides behave then as free rotators. In that region, there exists no ordered phase but only single ionic cyanides undergoing individual dynamical freezing. We call this state a single-ion state (see Fig. 4).

(ii) The other possibility is to assume equation (13) holds as well when $x<\bar{x}_{\mathrm{c}}$. The random distribution of volume differences has now turned the ferroelastic coupling into an antiferroelastic coupling, i.e., $G<0$ in equation (13). We thus obtain a new diluted antiferroelastic phase. The transition line is given by $-k T_{\mathrm{c}}$ from equation (15) in the concentration range $0<x<\bar{x}_{\mathrm{c}^{*}}$. The phase diagram associated with this situation is shown in figure 5 . Within the new ordered phase the ferroelastic order parameter is zero, i.e.

$$
\frac{1}{N} \sum_{i=1}^{N}\left\langle S_{l}\right\rangle=0,
$$

with however at each site

$$
\left\langle S_{i}\right\rangle \neq 0
$$

since there exists an antiferroelastic long range order. It is worth stressing that dilution can be misleading in making this ordered phase look like a glass. Here there exists no frustration. Moreover the associated transition is a true static phase transition. As a function of $x$ we have a re-entrant phenomenon at intermediate concentrations. 


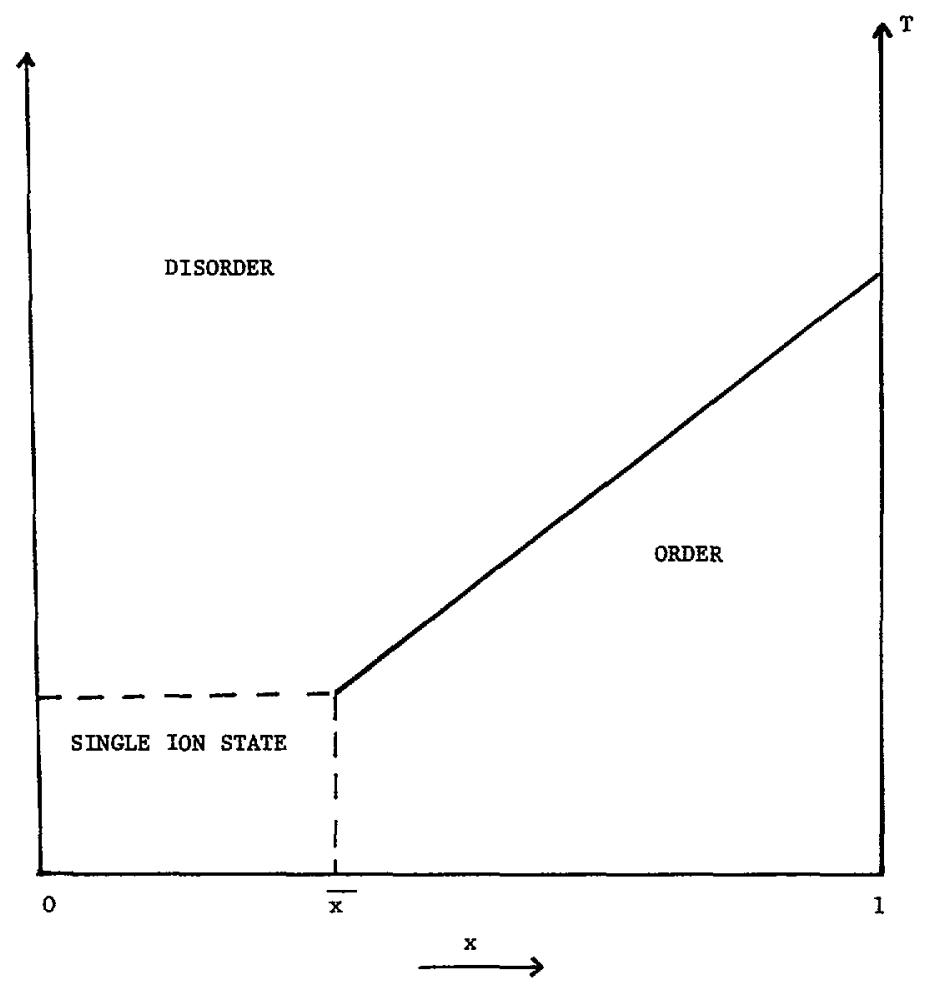

Fig. 4. - Phase diagram for case B-(i).

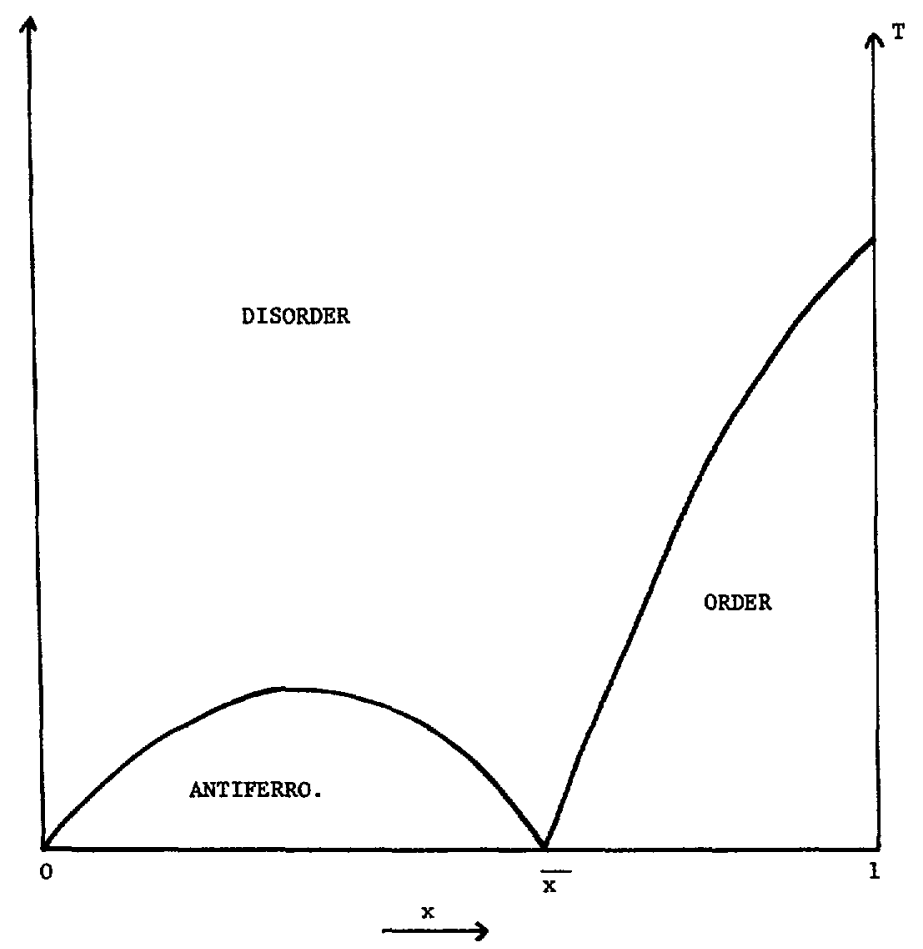

Fig. 5. - Phase diagram for case B-(ii). 


\section{Three possible phase diagrams.}

At this stage depending on physical characteristics of the system three phase diagrams are possible. If $\bar{x}_{c}<0$, we have case $A$ for which there exists one ordered ferroelastic phase. Dilution makes the transition temperature to decrease and eventually vanish at $x=0$ which is the mean field percolation threshold (Fig. 3). On the contrary, when $\bar{x}_{\mathrm{c}}>0$, we have case B for which two possibilities exist. For the first one (i), the phase diagram is similar to that of case A. But now the transition vanishes at $\bar{x}_{\mathrm{c}}$ thus creating a single-ion state (Fig. 4). On the opposite, possibility (ii) has an additional ordered phase in the range $x<\bar{x}_{\mathrm{c}}$. The corresponding ordering is antiferroelastic and the transition temperature vanishes also at $x=0$ like in case A (Fig. 5).

To select which phase diagram corresponds to $\mathrm{KCN}-\mathrm{KBr}$ mixtures requires an evaluation of numerical values of $J_{0}, J, \varphi$ and $h$. Due to the over-simplified character of our analysis, such evaluation is out of the scope of the present qualitative description. In the following we restrict the study to case A (Fig. 3), since up to now there exists no experimental support to the existence on an additional static phase transition into an antiferroelastic phase directly from the disordered phase.

\section{The broken percolation dressing.}

In case $\mathrm{A}$ the transition disappears at $x=0$ due to dilution. It is worth stressing however that equation (15) describes a non-linear percolation problem since $G$ depend on $x$ (Eq. (13)). However cancellation of the critical temperature is produced by products $\varepsilon_{i} \varepsilon_{j}$ appearing in equation (10). It is then natural to expect it to occur at the threshold of usual percolation. Going beyond mean field treatment, the exact percolation threshold for an fcc lattice is $p_{\mathrm{c}} \simeq 0.20$ [12]. Using that result the phase transition is lost at $p_{\mathrm{c}}=0.20$ instead of $p=0$ in figure 3 . In contrast experiments on $\mathrm{KNC}-\mathrm{KBr}$ mixtures found that the ferroelastic transition is lost at $x_{\mathrm{c}}=0.6[1]$.

Differences between $p_{\mathrm{c}}$ and actual threshold concentrations have been already found in other physical systems $[15,16]$. Such a discrepancy indicates that some cyanides do not participate in the propagation of long range order at low temperatures. It means that site percolation of cyanides should be discriminated from the corresponding orientational percolation. It is then natural to associate this effect with the presence of bromides and to assume that on the average some cyanides get trapped along some orientation which prevent them to participate in the propagation of long range orientational order. To account for such an effect we make a simple linear hypothesis for cyanide concentration. We assume an effective density of free cyanides with respect to orientational percolation

$$
x^{\prime}=x-\alpha(1-x)
$$

where $\alpha$ is the fraction of cyanides trapped on the average by a bromide.

A physical argument to justify such anisotropic effect could be the fact that locally the system is not cubic with spherical bromides not sitting at equal distance from their respective twelve neighbors [17]. However as the system remains macroscopically cubic at low temperatures, the pinning must be along random orientations to preserve overall cubic symmetry in the ensemble average.

A justification of the linear form used in equation (19) for the effective density of cyanides could be related to a recent experiment [4] which found a linear increase in $x$ for the hindering barriers of the orientational potential of cyanides.

Using equation (19) the ferroelastic transition is lost at a concentration $x_{c}$ such that

$$
p_{\mathrm{c}}=x_{\mathrm{c}}-\alpha\left(1-x_{\mathrm{c}}\right)
$$


giving

$$
x_{\mathrm{c}}=\frac{p_{\mathrm{c}}+\alpha}{1+\alpha}
$$

where $p_{\mathrm{c}}$ is the percolation threshold. At this stage a direct calculation of $\alpha$ is rather complicated [17]. Here we will use the experimental value $x_{c}=0.6$, to get from equation (21)

$$
\alpha=\frac{x_{\mathrm{c}}-p_{\mathrm{c}}}{1-x_{\mathrm{c}}}
$$

which gives $\alpha=1$ when $p_{\mathrm{c}} \simeq 0.2$ and $x_{\mathrm{c}} \simeq 0.6$.

Therefore each bromide traps on the average one cyanide. Site substitution of a dumbelllike shape cyanide with a spherical bromide results in local steric hindrances which pin at random one cyanide by freezing its orientation.

Given a bromide there exists twelve neighbor sites on the fcc anion sublattice occupied by $\mathrm{CN}$ or $\mathrm{Br}$ according to $x$ and $(1-x)$ respectively. In the present approach it is suggested that not all present cyanides among the twelve neighbors participate to the propagation of long range order. One of them will be blocked along a random orientation upon lowering temperature, before nearest neighbor cyanides start to order. From equations (19) and (22) the effective density of cyanides which participate in the establishment of orientational long range order is $x^{\prime}=2 x-1$. Within such a picture a saturation mechanism occurs when all cyanides get trapped by bromides, which happens when $x^{\prime}=0$ at

$$
x_{\mathrm{s}}=\frac{\alpha}{1+\alpha},
$$

giving $x_{\mathrm{s}}=0.5$ for $\alpha=1$.

\section{Phase diagram of $\mathrm{KCN}-\mathrm{KBr}$ mixtures.}

We are now in a position to present a qualitative description of the effects of dilution on the ferroelastic transition (see Fig. 2) as a function of cyanide concentration. The results are then compared to various experimental findings.

Upon lowering temperature from the disordered phase where cyanides behave as free rotators, there exists some temperature $T_{\mathrm{a}}$, associated with the activation energy of the trapped cyanides, higher than the transition temperature. At $T_{\mathrm{a}}$, trapped cyanides get blocked along random orientations while non-trapped cyanides are still free to reorient. However in the vicinity of the transition temperature, blocked cyanides behave as local random walls with respect to the establishment of long range orientation order among free cyanides. Nevertheless as long as there exists an infinite orientational cluster, i.e., for $x>0.6$, the ferroelastic transition occurs.

On the other hand for $x<0.6$ we can build the following picture. There is still a percolating lattice with respect to site percolation but not with respect to orientational percolation. Therefore, long range orientational order is not possible any longer and the ferroelastic transition is lost. The net result of such a broken percolation should be the formation of ferroelastic domains by free cyanides. These domains are expected to have a random distribution of orientations according to the random domain walls created by the trapped cyanides. Motion of domain walls is therefore expected in this description. With respect to the experimental situation a primary elastic loss peak in torsion pendulum experiments [3] has been indeed already interpreted in terms of ferroelastic domains. Moreover, the above description implies existence of ferroelastic domains also in the ordered ferroelastic phase. 
Very recent shear torque experiments did find a well developed primary elastic loss peak above $x_{\mathrm{c}}=0.6$, for $x<0.75$, in agreement with our approach [6].

Shear torque experiments also found a lower value $x_{\mathrm{s}} \simeq 0.5$ for a well defined primary loss peak [3], thus suggesting the existence of ferroelastic domains only in the range $x>0.5$. From equations (19) and (22) it is seen that ferroelastic domains created by free cyanides will shrink with decreasing cyanide concentration to eventually disappear at $x_{\mathrm{s}}=0.5$ which is exactly the experimental finding. Existence of domains in the range $0<x<0.6$ has been suggested previously using topological arguments [18].

In the concentration range $0.2<x<0.5$ we have an infinite site percolating cluster of cyanides. However each cyanide is pinned by bromides upon lowering temperature, which gives rise to a dynamical freezing. There exists indeed a competition between local steric hindrances which freeze individual cyanides and exchange interactions which favor collective freezing. The result at low temperature is what we call a collective classical glass. It is worth stressing that this glass has no connection to spin-glasses. In the present case there exists no phase transition, no frustration, and the freezing is a dynamical effect (see Fig. 2).

Below the percolation threshold $p_{c}=0.2$ we have single ionic cyanides which undergo individual dynamical freezing, producing a single-ion state.

\section{Comment on $\mathrm{KCN}-\mathrm{NaCN}$ mixtures.}

While $\mathrm{KCN}-\mathrm{KBr}$ mixtures have attracted much experimental work [1-6], less effort has been devoted to the study of $\mathrm{KCN}-\mathrm{NaCN}$ mixtures [5, 19]. The corresponding phase diagram is also expected to exhibit a glassy phase but only at intermediate range of alloying. Random strains generated by volume differences between cations $\mathrm{K}$ and $\mathrm{Na}$ are believed to be instrumental in the establishment of the glassy phase in a similar fashion as for $\mathrm{KCN}-\mathrm{KBr}$ mixtures. However we can already from the present study show that the glassy state in $\mathrm{KCN}-\mathrm{NaCN}$ mixtures is indeed of a different physical nature than for the corresponding glassy state found in $\mathrm{KCN}$ $\mathrm{KBr}$ mixtures.

Effects of volume differences can be accounted for as in part 2 with random strains given by equations (4), (5) and (6). The main difference is that now the $\left\{\varepsilon_{\imath}\right\}$ variables are not attached to rotational degrees of freedom but to cations $\mathrm{K}$ and $\mathrm{Na}$ on the interpenetrated fcc lattice. We have $\varepsilon_{i}=1$ for $\mathrm{K}$ and $\varepsilon_{l}=0$ for $\mathrm{Na}$. Since there exists no dilution of cyanides the Hamiltonian corresponding to $H_{\mathrm{R}}$ (Eq. (10)) is here

$$
\bar{H}_{\mathrm{R}}=-\sum_{\langle i, J\rangle} G_{i j} \mathbf{S}_{i} \mathbf{S}_{j}-\frac{J^{2}}{2 \varphi} \sum_{\langle, J\rangle} \mathbf{S}_{i}^{2} \mathbf{S}_{J}^{2},
$$

where $G_{i j}$ given still by equation (11), but not by equation (13), takes the values

$$
\begin{aligned}
& J_{0}-\frac{2 h_{\mathrm{L}} J}{\varphi}(1-x)+\frac{2 h_{\mathrm{S}} J}{\varphi}(1-x), \\
& J_{0}-\frac{2 h_{\mathrm{L}} J}{\varphi}(1-x)+\frac{h_{\mathrm{S}} J}{\varphi}(1-2 x), \\
& J_{0}-\frac{2 h_{\mathrm{L}} J}{\varphi}(1-x)-\frac{2 h_{\mathrm{S}} J}{\varphi} x,
\end{aligned}
$$

with respective probabilities, $x^{2}, 2 x(1-x)$, and $(1-x)^{2}$. From equations (25), (26) and (27) it is seen that random competing exchange interactions may now be obtained, depending on values of $J_{0}, h_{\mathrm{L}}, h_{\mathrm{S}}, J$ and $\varphi$. At this stage it appears that the nature of an eventual glass state in $\mathrm{KCN}-\mathrm{NaCN}$ could be indeed related to spin glass ordering on the contrary of $\mathrm{KCN}-\mathrm{KBr}$ case where couplings were found to be uniform (Eq. (13)). 
A detailed study of $\mathrm{KCN}-\mathrm{NaCN}$ mixtures is left for future work [20]. We can nevertheless conclude that the glassy phase is of a different physical nature in respectively $\mathrm{KCN}-\mathrm{KBr}$ and $\mathrm{KCN}-\mathrm{NaCN}$ mixtures. A connection to spin-glasses may exist for the last case, but not for the first one.

\section{Critical review of the RF model.}

The orientational random field model (ORFM) [9] is based on the use of random static strains generated by site fluctuations in the concentration of bromides. We first formulate the main features of the ORFM in the spirit of the Ising like language used in this paper. A critical analysis is then presented. The experimental support for the ORFM is discussed.

8.1 PRESENTATION OF THE ORFM. - The frame of the ORFM is a Hamiltonian of the form

$$
H_{\mathrm{ORFM}}=-\sum_{i} h_{i} u_{i}-\lambda \sum_{i} u_{i} \varepsilon_{i} S_{i}+\frac{1}{2} \varphi \sum_{i} u_{i}^{2}
$$

where $\lambda$ is a constant and

$$
h_{i}=h\left(\varepsilon_{i}-x\right) .
$$

First and last terms of $H_{\mathrm{ORFM}}$ are similar to those used in our model. The difference is the middle term which represents a bilinear displacement-rotation coupling. In reference [9] the Hamiltonian is cast in a more complicated form, but main features are identical. After various manipulations a coupling term is obtained in the form

$$
H_{\mathrm{c}}=-\sum_{i} \ell_{i} \varepsilon_{i} S_{i}
$$

where,

$$
\ell_{i}=\sum_{j} h_{i j}\left(\varepsilon_{j}-x\right)
$$

with $h_{\imath j}$ being a complicated function. From equation (31) it is found that

$$
\mathbb{P}_{i}=0
$$

and

$$
\mathbb{l}_{i}^{2} \propto x(1-x)
$$

where the bar denotes a configurational average on the $\left\{\varepsilon_{i}\right\}$. From equations (32) and (33) it is then stated [9] that the rotational problem can be mapped onto the well studied random field problem in magnetic systems [21]. It is then assumed that $\ell_{i}$ (Eq. (31)) has a Gaussian distribution [9] in analogy to the magnetic case [21].

8.2 CRITICAL REVIEW OF THE ORMF. - We now proceed on a critical analysis of the ORFM. First it should be stressed that from equation (30) the fields coupled to rotational degrees of freedom are indeed $\left\{\varepsilon_{i} \ell_{i}\right\}$ and not $\left\{\ell_{i}\right\}$. It is of importance since $\ell_{i}$ is a function of $\varepsilon_{i}$ (Eq. (31)). We thus have in particular the property

$$
\overline{\varepsilon_{\imath} \mathbb{\ell}_{i}}=h_{i i} x(1-x) \text {, }
$$


where the value of $h_{i i}$ is basic to determine the symmetry property of the fields which couple to rotations. A non-zero value of $h_{i t}$ would make the fields $\left\{\varepsilon_{i} \ell_{i}\right\}$ symmetry-breaking fields with respect to the $\left\{S_{i}\right\}$. Such fields would be then forbidden by the symmetry of $\mathrm{KCN}-\mathrm{KBr}$ mixtures which do exhibit a disordered phase at high temperatures. However a numerical calculation of $h_{i l}$ is difficult. It has been claimed that $h_{i 1}$ is indeed zero using both general symmetry arguments [22] and poor numerical estimates [23]. It is still an open question.

Moreover even if $h_{i i}$ was indeed zero, the hypothesis of a Gaussian distribution made in reference [9] for the $\left\{\ell_{i}\right\}$ is not proven. At this stage in contradistinction to that Gaussian assumption, we argue that the distribution of the $\left\{\ell_{i}\right\}$ cannot be symmetric.

The starting physical ingredient of the problem is the random distribution of $\left\{\varepsilon_{i}\right\}$ where $\varepsilon_{i}=1$ with probability $x$ and $\varepsilon_{i}=0$ with probability $(1-x)$. This distribution is not symmetric expect at the special case $x=1 / 2$. Therefore within the present framework any manipulation must preserve the non-symmetric character of the quenched random disorder making a Gaussian distribution not compatible with the problem. To conclude, the $\left\{\ell_{i}\right\}$ within the ORFM must have a non-symmetric distribution.

Moreover a non-symmetric distribution of the $\left\{\boldsymbol{\ell}_{i}\right\}$ would indeed break the symmetry of rotational degrees of freedom, thus suppressing the transition to a disordered phase at high temperatures. However from experiment the existence of a disordered phase high temperatures is well established, as well as a transition into it, for $1<x<0.6$.

On this basis we conclude that the ORFM [9] is not allowed by the symmetry of $\mathrm{KCN}-\mathrm{KBr}$ mixtures.

8.3 EXPERIMENTAL SUPPORT FOR THE ORFM. - The most detailed analysis of experimental results in term of the ORFM was done in a study of static-shear elasticity in $\mathrm{RbCN}-\mathrm{RbBr}$ mixtures [24]. However in reference [24] all the formalism used comes from the magnetic random field problem [21] which does have a symmetric distribution, although the physical motivation is based on the ORFM model of reference [9]. The difficulty is thus twofold :

(i) The ORFM [9] does not describe the same physical situation as the magnetic RFM [21]. Although both models have a random field distribution with a zero configurational average, the first one [9] has symmetry-breaking fields, while the second one uses non-symmetrybreaking fields [21].

(ii) A magnetic RFM seems to fit some experimental data [24]. However, it is a totally adhoc model. There exists no physical justification to the use of random fields with a symmetric distribution. Along these lines it can be noticed that ad-hoc random fields have already been used to describe pure plastic crystals like $\mathrm{KCN}$ [25]. At this stage the problem is to find a physical mechanism to explain the existence of these random fields, if any.

\section{Conclusion.}

We have been able to build a sketch of the full phase diagram of $\mathrm{KCN}-\mathrm{KBr}$ mixtures (Fig. 2). The idea of broken percolation which results from the discrimination of site percolation from orientational percolation, has been instrumental to derive our results. In case A (see part 3), two regions were found. For $0.6<x<1$, a phase transition occurs into a ferroelastic phase. On the opposite, for $x<0.6$, a glass phase is found with no static phase transition. The glass has a domain structure for $0.5<x<0.6$. It then turns to a collective glass when $0.2<x<0.5$, to finally end as a single ion state for $x<0.2$. Our findings are consistent with various experiments and suggest a unified explanation of the complex phase diagram of $\mathrm{KCN}$ KBR mixtures.

It is worth noticing that the present approach holds for $\mathrm{KCN}-\mathrm{KCl}$ mixtures as well. In that case we get $\alpha=3$ from equation (23). The phase diagram is identical to figure 2, with now 
$x_{\mathrm{c}}=0.8, x_{\mathrm{d}}=0.75$ and $p_{\mathrm{c}}=0.2$. The domain region is reduced by a factor 2 in the cyanide concentration.

While neither competing interactions nor orientational random fields were found for $\mathrm{KCN}$ $\mathrm{KBr}$ mixtures, it was shown that the glass state of $\mathrm{KCN}-\mathrm{NaCN}$ mixtures may be associated to random competing interactions.

At the present stage, more experimental and numerical investigations would be helpful to further clarify our theoretical proposal.

Note added in proofs:

A recent Preprint by A. Loidl reported on a phase diagram, similar to figure 2, constructed from single crystal neutron diffraction experiments.

\section{Acknowledgements.}

I would like to thank P. Doussineau, A. Ghazali, K. Knorr, A. Levelut, and S. R. Salinas for very helpful discussions.

\section{References}

[1] Rowe J. M., Rush J. J., Hinks D. G. and Susman S., Phys. Rev. 43 (1979) 1158. KNORR K., Phys. Scr. T19 (1987) 531.

[2] KNORR K. and LOIDl A., Phys. Rev. B 57 (1985) 5387.

[3] KNorr K., Volkmann U. G. and Loidl A., Phys. Rev. Lett. 57 (1986) 2544.

[4] Mertz B., Bohmer R., Eisele B. and Loidl A., Z. Phys. B Cond. Matt. 79 (1990).

[5] Loidl A., Ann. Rev. Phys. Chem. 40 (1989) 29.

[6] Hessinger J. and KnorR K., Phys. Rev. Lett. 63 (1989) 2749.

[7] Lewis L. J. and Klein M. L., J. Phys. Chem. 91 (1987) 4990 ; Phys. Rev. B 40 (1989) 7080.

[8] Michei K. H. and Naudt J., Phys. Rev. Lett. 39 (1977) 212.

[9] Michel K. H., Phys. Rev. B 35 (1987) 1405.

[10] Galam S., J. Appl. Phys. 67 (1990) 5979.

[11] Krivoglaz M. A., Théorie de la diffusion des rayons $\mathrm{X}$ et des neutrons thermiques par les cristaux réels (Masson, Paris, 1969).

[12] Stauffer D., Phys. Rept. 54 (1979) 1.

[13] Salinas S. R., J. Phys. C 7 (1974) 241.

[14] Galam S., Henriques V. B. and Salinas S. R., Phys. Rev. B 42 (1990) 6720.

[15] Adler J., Palmer R. G. and Meyer H., Phys. Rev. Lett. 58 (1987) 882.

[16] Coutinho S., Albino J., de Aguiar O., Brady Moreira F. G. and de Almeida J. R. L., Phys. Rev. B 36 (1987) 8478.

[17] Galam S. and DePONDT P., to be published.

[18] IHM J., Phys. Rev. B 31 (1985) 1674.

[19] Schrader T., Loidl A. and Vogt T., Z. Phys. B 79 (1990) 423.

[20] Galam S. and Salinas S. R., to be published.

[21] Aharony A., Phys. Rev. B 18 (1978) 3318.

[22] PICK R. M., Private communication.

[23] Bostoen C. and Mrchel K. H., Private communication.

[24] Fossum J. O., Wells A. and Garland C. W., Phys. Rev. B 38 (1988) 412.

[25] Galam S., Phys. Lett. A 122 (1987) 271. 